\title{
Reentrant and forward phase diagrams of the anisotropic three-dimensional Ising spin glass
}

\author{
Can Güven, ${ }^{1}$ A. Nihat Berker, ${ }^{1,2,3}$ Michael Hinczewski, ${ }^{3}$ and Hidetoshi Nishimori ${ }^{4}$ \\ ${ }^{1}$ Department of Physics, Koç University, Sarlyer 34450, Istanbul, Turkey \\ ${ }^{2}$ Department of Physics, Massachusetts Institute of Technology, Cambridge, Massachusetts 02139, USA \\ ${ }^{3}$ Feza Gürsey Research Institute, TÜBITAK-Bosphorus University, Çengelköy 34680, Istanbul, Turkey \\ ${ }^{4}$ Department of Physics, Tokyo Institute of Technology, Oh-okayama, Meguro-ku, Tokyo 152-8551, Japan
}

(Received 6 February 2008; published 9 June 2008)

\begin{abstract}
The spatially uniaxially anisotropic $d=3$ Ising spin glass is solved exactly on a hierarchical lattice. Five different ordered phases, namely, ferromagnetic, columnar, layered, antiferromagnetic, and spin-glass phases, are found in the global phase diagram. The spin-glass phase is more extensive when randomness is introduced within the planes than when it is introduced in lines along one direction. Phase diagram cross sections, with no Nishimori symmetry, with Nishimori symmetry lines, or entirely imbedded into Nishimori symmetry, are studied. The boundary between the ferromagnetic and spin-glass phases can be either reentrant or forward, that is either receding from or penetrating into the spin-glass phase, as temperature is lowered. However, this boundary is always reentrant when the multicritical point terminating it is on the Nishimori symmetry line.
\end{abstract}

DOI: 10.1103/PhysRevE.77.061110

PACS number(s): 75.10.Nr, 64.60.aq, 05.70.Fh, 05.10.Cc

\section{INTRODUCTION}

The Ising spin glass [1] yields a phase diagram with a distinctively complex ordered phase, in $d=3$. A wide accumulation of methods and results has occurred for this system. Most remarkably, in spite of its high spatial dimension and complex ordering behavior, exact or precise information is being obtained for this system [2-12]. Thus, in the phase diagram in terms of temperature and concentration of antiferromagnetic bonds, the occurrence of the Nishimori symmetry line has been deduced $[2,3]$ and the accurate location of the multicritical point has been predicted $[10,12]$. Furthermore, in systems with the Nishimori symmetry, it has been shown that the ferromagnetic phase cannot extend to antiferromagnetic bond concentrations beyond that of the multicritical point $[2,3]$. The two remaining options being a straight line or a reentrance situation, subsequent works
$[19,20]$ on hierarchical lattices have shown that for these systems, the spin-glass phase diagram is reentrant, namely, that below the multicritical point, the ferromagnetic phase recedes from the spin-glass phase as temperature is lowered. Exact results recently have also been extended to Potts spin glasses [21]. These results complement recent precise calculations, using Monte Carlo simulations, on cubic lattices [13-18].

A spatially uniaxially anisotropic $d=3$ system is studied in this work, to our knowledge the first study of quenched randomness and frustration in a spatially anisotropic higherdimensional system. In fact, both anisotropy and quenched randomness have acquired increased relevance from hightemperature superconductivity results $[22,23]$. Our calculation is exact for a hierarchical lattice and approximate for a cubic lattice. We find a rich phase diagram (e.g., Fig. 1) with five different ordered phases, namely with ferromagnetic, an-

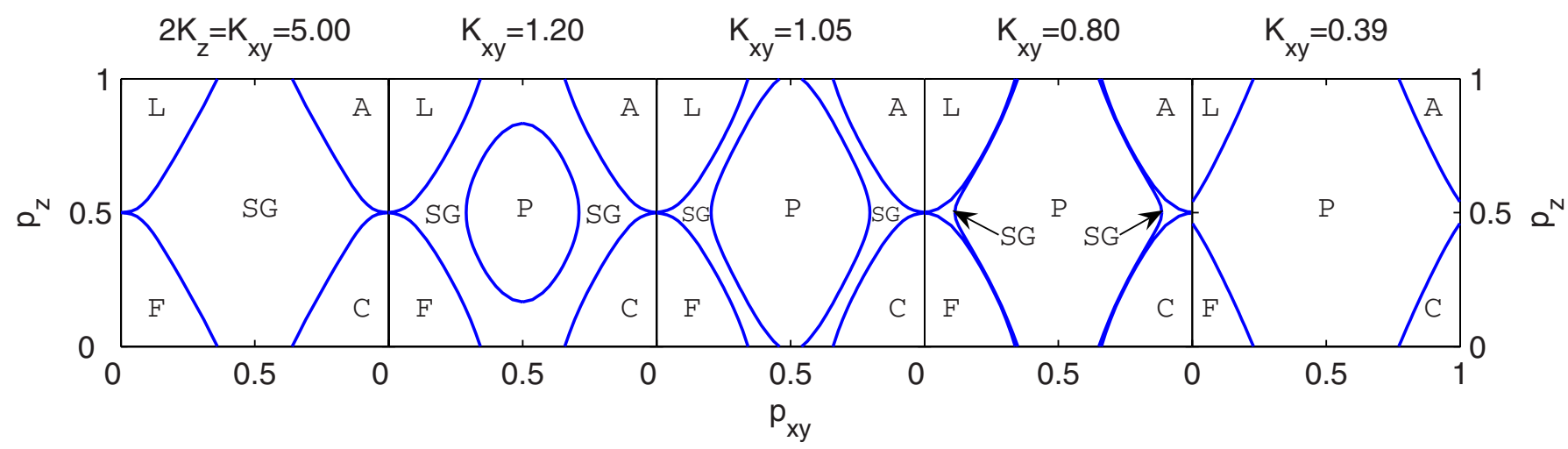

FIG. 1. (Color online) Constant-temperature cross sections of the global phase diagram for $K_{z} / K_{x y}=0.5$, as a function of $p_{x y}$ and $p_{z}$, which are the concentrations of antiferromagnetic $x y$ and $z$ bonds, respectively. At low temperatures (high $\left.K_{x y}\right)$, the central spin-glass (SG) phase separates the corner ferromagnetic (F), columnar (C), antiferromagnetic (A), and layered (L) phases. The diagrams are twofold symmetric along each axis, but not fourfold symmetric, due to the difference between longitudinal $\left(p_{x y}=0\right)$ and transverse $\left(p_{z}=0\right)$ spin glasses. As temperature increases, the paramagnetic $(\mathrm{P})$ phase appears at the central point, first reaches the transverse spin-glass system and eliminates the spin-glass phase, then reaches the longitudinal spin-glass system and eliminates the spin-glass phase. In the latter system, the spin-glass and paramagnetic phases simultaneously occur for a very narrow range of temperatures, as also seen in the inset in the lower left panel of Fig. 3. 

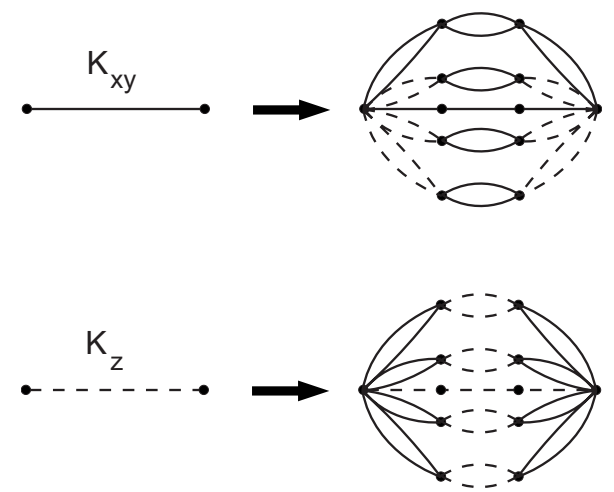

FIG. 2. Construction of the uniaxially anisotropic $d=3$ hierarchical model. Two graphs are mutually and repeatedly selfimbedded. Note that for $K_{x y}=0, K_{z}=0$, and $K_{x y}=K_{z}$, the system reduces, respectively, to the $d=1$, isotropic $d=2$, and isotropic $d$ $=3$ systems.

tiferromagnetic, layered, columnar, and spin-glass order. The spin-glass phase is more extensive when randomness is introduced within the planes than when it is introduced in lines along one direction.

The global phase diagram includes cross sections with no Nishimori symmetry, cross sections with Nishimori symmetry lines, and a cross section entirely imbedded within Nishimori symmetry. Thus, the multicritical point between the spin-glass, ferromagnetic, and paramagnetic phases, previously found to occur on the Nishimori symmetry line, is also found here at points with no Nishimori symmetry, but renormalizes to a fixed distribution of interaction probabilities that obeys Nishimori symmetry. Nevertheless, we find that the boundary between the ferromagnetic and spin-glass phases can be either reentrant or forward, that is either receding from or penetrating into the spin-glass phase, as temperature is lowered. When the multicritical point is not on the Nishimori symmetry line, the ferromagnetic-spin glass boundary can be reentrant or forward. However, when the multicritical point is on the Nishimori symmetry line, this boundary is always reentrant $[19,20]$, consistently with the rigorous result $[2,3]$.

\section{UNIAXIALLY ANISOTROPIC SPIN GLASS}

The uniaxially anisotropic Ising spin-glass system has the Hamiltonian

$$
-\beta H=\sum_{u} \sum_{\langle i j\rangle_{u}} K_{i j}^{u} s_{i} s_{j},
$$

where $s_{i}= \pm 1$ at each site $i,\langle i j\rangle_{u}$ denotes a sum over nearestneighbor pairs of sites along the $z$ direction $(u=z)$ or in the $x y$ plane $(u=x y)$, and the bond strengths $K_{i j}^{u}$ are equal to $K_{u}>0$ with probability $1-p_{u}$ and $-K_{u}$ with probability $p_{u}$, respectively corresponding to ferromagnetic and antiferromagnetic interaction. When imbedded in a cubic lattice, the Hamiltonian (1) yields a uniaxially anisotropic $d=3$ system.

Hierarchical lattices are $d$-dimensional lattices yielding exact renormalization-group solutions to complex statistical mechanics problems. These lattices are constructed by the repeated self-imbedding of a graph into a bond [24-26]. The shortest path between the external vertices of the graph gives the length rescaling factor $b$ and the number of bonds in the graph gives the volume rescaling factor $b^{d}$, from which the dimension $d$ is determined. Hierarchical lattices have been used to study a wide variety of problems, including chaotic rescaling [27,28], spin-glass [19], random-field [29], Schrödinger equation [30], lattice-vibration [31], dynamic scaling [32], random-resistor network [33], aperiodic magnet [34], complex phase diagram [35], directed-path [36,37], heteropolymer [38], directed-polymer [39], and, most recently, scale-free and small-world network [40-47] systems, etc. More recently, hierarchical lattices have been created [48] for the study of spatially anisotropic systems. The mutual repeated self-imbedding of two appropriately chosen graphs, with differentiated interactions, yields a uniaxially anisotropic system, whereas a higher number of graphs is needed to achieve higher spatial anisotropy [48]. These hierarchical systems must reduce to isotropy and/or lower spatial dimensions when corresponding interactions are set equal to each other or to zero, as illustrated in Fig. 2. An anisotropic hierarchical lattice has already been used to obtain the phase diagram of the uniaxially anisotropic $d=3 t J$ model of electronic conduction [22]. When imbedded into the hierarchical lattice of Fig. 2, the Hamiltonian (1) yields a uniaxially anisotropic $d=3$ spin-glass system that is exactly soluble.

\section{EXACT RENORMALIZATION-GROUP SOLUTION: FLOWS OF THE QUENCHED DISTRIBUTIONS OF THE ANISOTROPIC SPIN-GLASS INTERACTIONS}

The renormalization-group solution proceeds in the direction opposite to the construction of a hierarchical model. Each graph is replaced by a renormalized bond via summation over the spins on the internal sites of the graph. This is achieved by a combination of two types of steps: the replacement, by a single bond $\widetilde{K}_{i j}$, of two bonds that are either in parallel, referred to as bond moving:

$$
\tilde{K}_{i j}=K_{i j}^{\mathrm{I}}+K_{i j}^{\mathrm{II}},
$$

or in series, referred to as decimation:

$$
\tilde{K}_{i k}=\frac{1}{2} \ln \left[\frac{\cosh \left(K_{i j}+K_{j k}\right)}{\cosh \left(K_{i j}-K_{j k}\right)}\right] .
$$

The quenched probability distribution $\widetilde{\mathcal{P}}(\widetilde{K})$ of the replacing bond is calculated by the convolution

$$
\tilde{\mathcal{P}}(\tilde{K})=\int d K^{\mathrm{I}} d K^{\mathrm{II}} \mathcal{P}_{\mathrm{I}}\left(K^{\mathrm{I}}\right) \mathcal{P}_{\mathrm{II}}\left(K^{\mathrm{II}}\right) \delta\left(\tilde{K}-R\left(K^{\mathrm{I}}, K^{\mathrm{II}}\right)\right),
$$

where $R\left(K^{\mathrm{I}}, K^{\mathrm{II}}\right)$ is the right-hand side of Eq. (2) and (3), $K^{\mathrm{I}}$ and $K^{\mathrm{II}}$ are the interactions entering the right-hand side of either of these equations, with quenched probability distributions $\mathcal{P}_{\mathrm{I}}\left(K^{\mathrm{I}}\right)$ and $\mathcal{P}_{\mathrm{II}}\left(K^{\mathrm{II}}\right)[19,29]$.

Accordingly, the renormalization of $\mathcal{P}_{x y}$ is obtained as follows, following the upper Fig. 2 in the direction opposite to the arrow: (i) from the bond-moving of $\mathcal{P}_{x y}$ with itself, obtaining $\widetilde{\mathcal{P}}_{1}$; (ii) from the bond-moving of $\mathcal{P}_{z}$ with itself, ob- 
TABLE I. Sinks of the renormalization-group flows in the different phases. These sinks are characterized here in terms of the average positive and negative interactions of their limiting quenched probability distribution.

\begin{tabular}{ccccc}
\hline \hline Phase & $\left\langle K_{+}^{x y}\right\rangle$ & $\left\langle K_{-}^{x y}\right\rangle$ & $\left\langle K_{+}^{z}\right\rangle$ & $\left\langle K_{-}^{z}\right\rangle$ \\
\hline Ferro & $+\infty$ & 0 & $+\infty$ & 0 \\
Antiferro & 0 & $-\infty$ & 0 & $-\infty$ \\
Columnar & 0 & $-\infty$ & $+\infty$ & 0 \\
Layered & $+\infty$ & 0 & 0 & $-\infty$ \\
Spin Glass & $+\infty$ & $-\infty$ & $+\infty$ & $-\infty$ \\
Para & 0 & 0 & 0 & 0 \\
\hline \hline
\end{tabular}

taining $\widetilde{\mathcal{P}}_{2}$; (iii) from the decimation of $\widetilde{\mathcal{P}}_{1}$ and $\widetilde{\mathcal{P}}_{1}$, obtaining $\widetilde{\mathcal{P}}_{3}$; (iv) from the decimation of $\widetilde{\mathcal{P}}_{2}$ and $\widetilde{\mathcal{P}}_{1}$, obtaining $\widetilde{\mathcal{P}}_{4}$; (v) from the decimation of $\mathcal{P}_{x y}$ and $\mathcal{P}_{x y}$, obtaining $\widetilde{\mathcal{P}}_{5}$; (vi) from the decimation of $\widetilde{\mathcal{P}}_{3}$ and $\widetilde{\mathcal{P}}_{1}$, obtaining $\widetilde{\mathcal{P}}_{6}$; (vii) from the decimation of $\widetilde{\mathcal{P}}_{4}$ and $\widetilde{\mathcal{P}}_{2}$, obtaining $\widetilde{\mathcal{P}}_{7}$; (viii) from the decimation of $\tilde{\mathcal{P}}_{5}$ and $\mathcal{P}_{x y}$, obtaining $\widetilde{\mathcal{P}}_{8}$; (ix) from the bondmoving of $\tilde{\mathcal{P}}_{6}$ and $\widetilde{\mathcal{P}}_{7}$, obtaining $\widetilde{\mathcal{P}}_{9}$; (x) from the bondmoving of $\widetilde{\mathcal{P}}_{7}$ and $\widetilde{\mathcal{P}}_{7}$, obtaining $\widetilde{\mathcal{P}}_{10}$; (xi) from the bondmoving of $\widetilde{\mathcal{P}}_{9}$ and $\widetilde{\mathcal{P}}_{10}$, obtaining $\widetilde{\mathcal{P}}_{11}$ (xii) finally, from the bond-moving of $\widetilde{\mathcal{P}}_{11}$ and $\widetilde{\mathcal{P}}_{8}$, obtaining the renormalized quenched distribution $\mathcal{P}_{x y}^{\prime}$. Thus, in each renormalizationgroup step, the renormalized distribution $\mathcal{P}_{x y}^{\prime}$ is obtained from the convolutions of 27 unrenormalized distributions $\mathcal{P}_{x y}$ and $\mathcal{P}_{z}$. The renormalized distribution $\mathcal{P}_{z}^{\prime}$ is similarly obtained from the convolutions of 27 unrenormalized distributions $\mathcal{P}_{x y}$ and $\mathcal{P}_{z}$, but with a different sequencing dictated by the lower Fig. 2.

The renormalization-group transformations of the quenched probability distributions $\mathcal{P}_{x y}$ and $\mathcal{P}_{z}$, given in the preceding paragraph, are implemented numerically, resulting in a distribution of interaction-strength values and a probability associated with each value, namely, a histogram. Thus, the initial $\pm K_{u}$ double- $\delta$ distribution functions, described after Eq. (1), are of course not conserved under the scale coarsening of the renormalization-group transformation. The number of histograms increases after each convolution. When a maximum number of histograms, set by us, is reached, a binning procedure is applied [19,29]: Before each convolution, the range of interaction values is divided into bins, separately for positive and negative interactions. The interactions falling into the same bin are combined according to their relative probabilities. The convolution then restores the set maximum number of histograms. In this work, we have used the maximum number of 90000 for histograms for each distribution $\mathcal{P}_{x y}$ and $\mathcal{P}_{z}$.

\section{PHASE DIAGRAMS AND FIXED DISTRIBUTIONS}

We have obtained the global phase diagram of the uniaxially anisotropic $d=3$ spin-glass system in terms of the original interactions and probabilities $\left(K_{x y}, K_{z}, p_{x y}, p_{z}\right)$. In each thermodynamic phase, quenched probability distributions

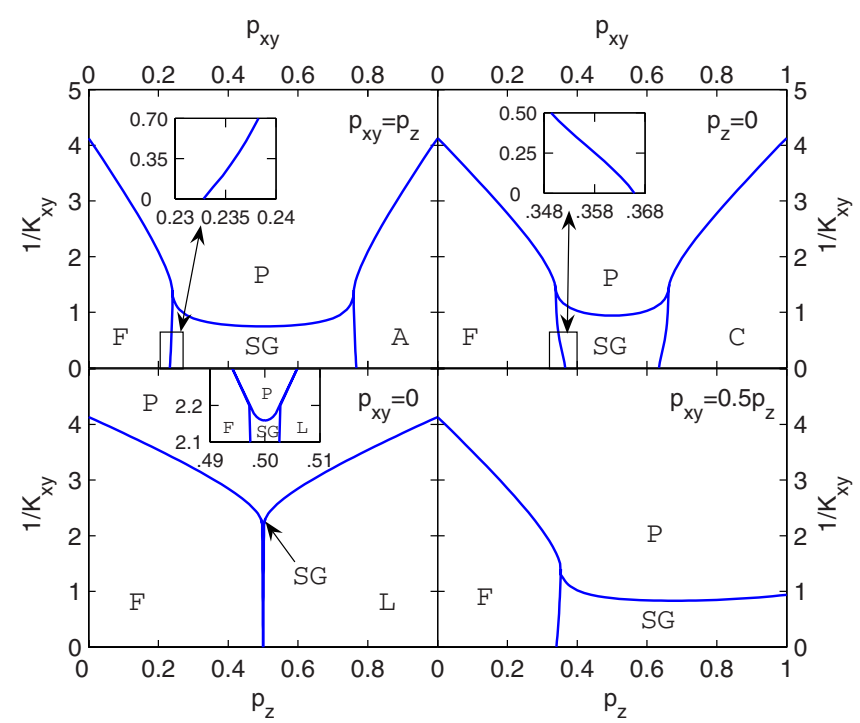

FIG. 3. (Color online) Temperature-concentration phase diagrams for isotropically mixed (upper left), transverse (upper right), longitudinal (lower left), and $p_{x y}=0.5 p_{z}$ spin-glass systems. In all cases, $K_{z} / K_{x y}=0.5$. The upper left and right phase diagrams are seen to be, respectively, reentrant and forward, namely, with a ferromagnetic phase that, respectively, recedes from or proceeds toward the spin-glass phase as temperature is lowered, as clearly seen in the insets. There are no points obeying Nishimori symmetry in the phase diagrams of this figure. Note the remarkably narrow spinglass phase, reaching zero temperature, in the longitudinal spinglass system, as also seen in the inset. All phase transitions in this figure are second order.

flow, under repeated renormalization-group transformations, to a limiting behavior (sink) characteristic of that thermodynamic phase. Phase boundary points flow to their own characteristic (unstable) fixed distributions, shown below. Analysis at these unstable fixed distributions yields the order of the phase transitions [19,29].

We find six different phases for this system, with corresponding sinks characterized in Table I in terms of the average positive and negative interactions of the limiting distribution. These phases are the ferromagnetic, antiferromagnetic, layered, columnar, spin-glass ordered phases and the disordered paramagnetic phase. In the layered phase, the spins are mutually aligned in each $x y$ plane; these planes of mutually aligned spins form an antiferromagnetic pattern along the $z$ direction. In the columnar phase, the spins are mutually aligned along the $z$ direction; these lines of mutually aligned spins form an antiferromagnetic pattern along the $x y$ directions. Both of these phases are thus distinct from the antiferromagnetic phase, which is antiferromagnetic in all three directions. There is a single spin-glass phase, extending to anisotropic systems.

\section{A. Phase diagrams with no Nishimori symmetry}

Cross sections of the global phase diagram are given in Figs. 1, 3, and 4. All phase transitions in these figures are second order. Figure 1 shows constant-temperature cross sections of the global phase diagram as a function of $p_{x y}$ and $p_{z}$. 


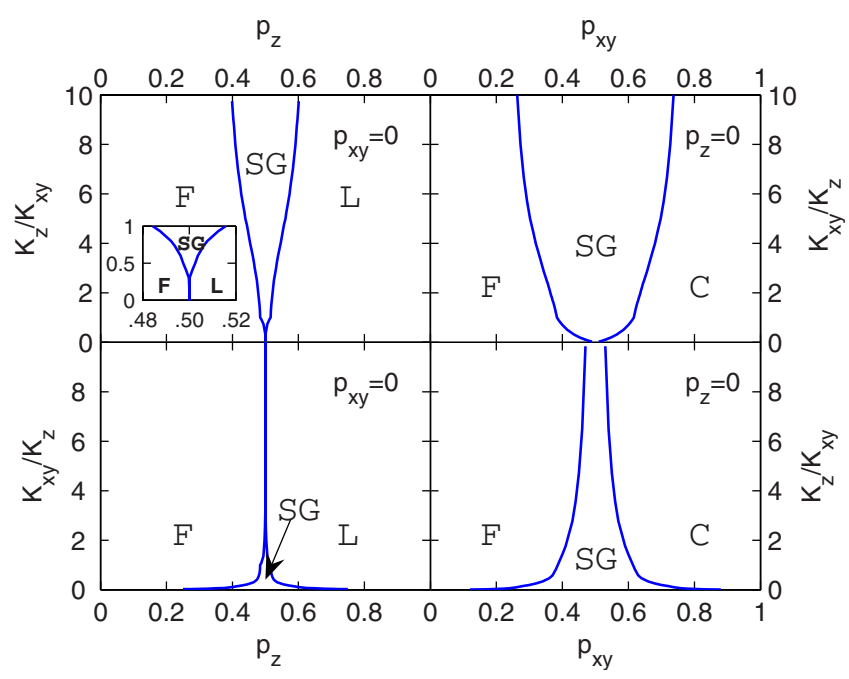

FIG. 4. (Color online) Zero-temperature phase diagrams of the longitudinal (left column) and transverse (right column) spin-glass systems. With the appropriate reversal in variables, the transverse and longitudinal spin-glass phase diagrams are seen here to be qualitatively similar, but quantitatively different. The spin-glass phase is more extensive in the transverse case. All phase transitions in this figure are second order.

At low temperatures (high $K_{x y}$ ), the central spin-glass (SG) phase separates the corner ferromagnetic $(\mathrm{F})$, columnar $(\mathrm{C})$, antiferromagnetic (A), and layered (L) phases. The diagrams are twofold symmetric along each axis, but not fourfold symmetric, due to the difference between transverse $\left(p_{z}=0\right)$ and longitudinal $\left(p_{x y}=0\right)$ spin glasses. As temperature increases, the paramagnetic $(\mathrm{P})$ phase appears at the central point, first reaches the transverse spin-glass system and eliminates the spin-glass phase, then reaches the longitudinal spin-glass system and eliminates the spin-glass phase. Figure 3 shows temperature-concentration phase diagrams for isotropically mixed, transverse, longitudinal, and $p_{x y}=0.5 p_{z}$ spin-glass systems. The upper left and right phase diagrams are seen to be, respectively, reentrant and forward, namely, with a ferromagnetic phase that, respectively, recedes from or proceeds toward the spin-glass phase as temperature is lowered, as clearly seen in the insets. The Nishimori symmetry (see below) is obeyed only at four isolated ordinary points in each cross section in Fig. 1 and is not obeyed at any point in the phase diagrams in Figs. 3 and 4, so that the forward behavior is not excluded by the rigorous results $[2,3]$.

A remarkably narrow spin-glass phase, reaching zero temperature, occurs in the longitudinal spin-glass system. Zerotemperature phase diagrams are shown in Fig. 4 for the longitudinal (left column) and transverse (right column) spinglass systems. With the appropriate reversal in variables, the longitudinal and transverse spin-glass phase diagrams are seen in this figure to be qualitatively similar, but quantitatively different. The spin-glass phase is more extensive in the transverse case. This can be understood from the more extensive intermixing of the ferromagnetic and antiferromagnetic bonds.
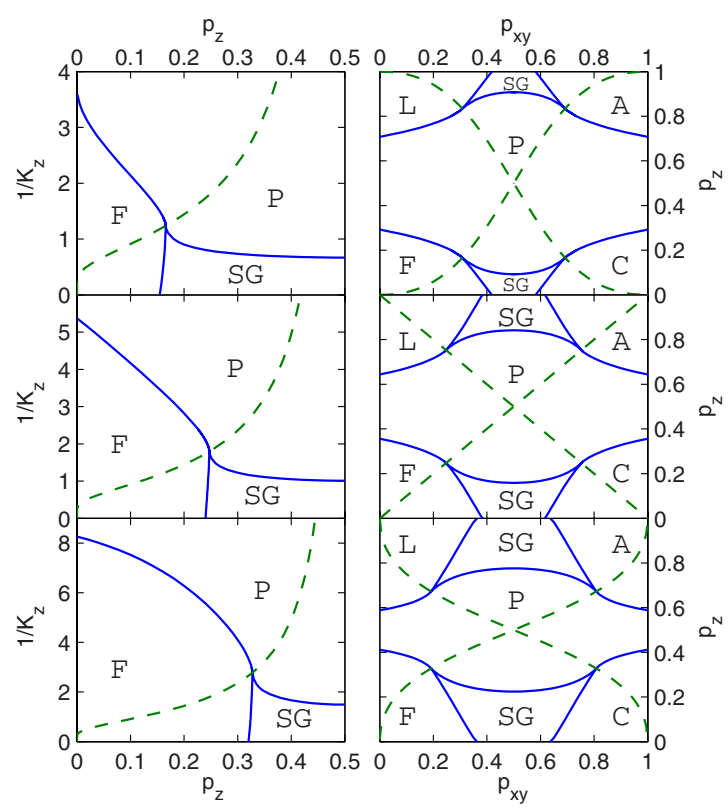

FIG. 5. (Color online) Phase diagrams with Nishimori symmetry lines (dashed) for different anisotropy parameters: The ratio $K_{z} / K_{x y}$ is 2,1 , and 0.5 from top to bottom. In the left column, $p_{x y}$ satisfies the Nishimori condition. In the right column, $K_{z}$ satisfies the Nishimori condition. All phase transitions in this figure are second order.

\section{B. Temperature-concentration phase diagrams with Nishimori symmetry curved lines}

The Nishimori symmetry condition [2,3] for isotropic systems

$$
\frac{1-p}{p}=e^{ \pm 2 K}
$$

generalizes, for uniaxially anisotropic spin-glass systems, to

$$
\frac{1-p_{x y}}{p_{x y}}=e^{ \pm 2 K_{x y}} \quad \text { and } \quad \frac{1-p_{z}}{p_{z}}=e^{ \pm 2 K_{z}} .
$$

For Nishimori symmetry to obtain, both equations have to be satisfied, but the signs in the exponents can be chosen independently. The Nishimori condition, in its general form

$$
\frac{\mathcal{P}_{u}\left(-K_{u}\right)}{\mathcal{P}_{u}\left(K_{u}\right)}=e^{ \pm 2 K_{u}}
$$

for each histogram pair of each distribution, is invariant (closed) under our renormalization-group transformation.

If one of the two conditions in Eq. (6) is fixed, phase diagram cross sections are obtained, in which Nishimori symmetry holds along a line. Thus, throughout the three phase diagrams on the left in Fig. 5, the condition on $\left(K_{x y}, p_{x y}\right)$ is fixed. The condition on $\left(K_{z}, p_{z}\right)$, and therefore Nishimori symmetry, is satisfied along the dashed lines on the left in Fig. 5. In these temperature versus concentration phase diagrams, it is seen that the multicritical points between the ferromagnetic, spin-glass, and paramagnetic phases lie on the Nishimori symmetry line. Furthermore, it has been proven $[2,3]$ that a forward phase diagram cannot occur below such a multicritical point that is on the symme- 


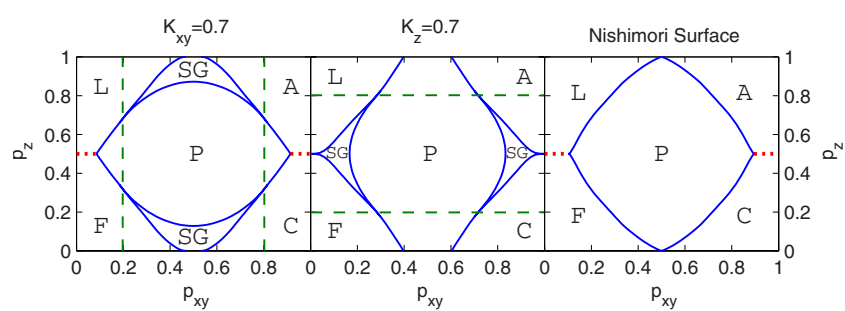

FIG. 6. (Color online) The Nishimori condition for $K_{z}$ is held throughout the leftmost figure and for $K_{x y}$ throughout the center figure. The complimentary Nishimori condition, for $K_{x y}$ and $K_{z}$ respectively, is held along the dashed straight lines, which intersect the ordered (F, L, A, or C)-spin-glass-paramagnetic multicritical points. In the rightmost figure both conditions are satisfied throughout the figure. In this figure, the phase boundaries around the paramagnetic phase are actually lines of the multicritical points where the paramagnetic, ordered (F, L, A, or C), and spin-glass (not seen in this cross section) phases meet. In the side figures, first-order boundaries (dotted) occur between the ferromagnetic and layered phases, and between the antiferromagnetic and columnar phases, terminating at $d=2$ critical points. All other phase transitions (full lines) in this figure are second order.

try line. On the left in Fig. 5, this is indeed the case, with reentrant phase diagrams, as also seen in isotropic spin glasses $[19,20]$. Recall that in Sec. IV A, multicritical points, between the same phases as here, that do not lie on Nishimori symmetry occur with both reentrant and forward phase diagrams. However, the latter nonsymmetric multicritical points flow, under renormalization-group transformations, to the (doubly unstable) fixed distribution of the symmetric multicritical points, therefore being in the same universality class and having the same critical exponents.

In the three phase diagrams on the right of Fig. 5, the condition on $\left(K_{z}, p_{z}\right)$ is fixed. In these concentrationconcentration phase diagrams, the multicritical points between the ordered (ferromagnetic, antiferromagnetic, layered, or columnar), spin-glass, and paramagnetic phases again lie on the Nishimori symmetry lines.

\section{Concentration-concentration phase diagrams with Nishimori symmetry straight lines}

In the phase diagrams in Fig. 5 , the ratio $K_{z} / K_{x y}$ is held constant. On the left and center of Fig. 6, again the condition in Eq. (6) on one interaction is fixed and the other interaction strength is held constant. Thus, the Nishimori symmetry lines become straight lines. The multicritical points between the ordered (ferromagnetic, antiferromagnetic, layered, or columnar), spin-glass, and paramagnetic phases again lie on the Nishimori symmetry lines. In the left phase diagram, due to the enforced Nishimori symmetry condition, $K_{z}=0$ along the line $p_{z}=0.5$ and the system reduces to $d=2$. Along this line, first-order transitions between ferromagnetic and layered phases and between antiferromagnetic and columnar phases terminate at $d=2$ critical points. From $p_{z} \neq 0.5, d=3$ secondorder boundaries between each ordered phase and the paramagnetic phase terminate on the $d=2$ critical points. In the center phase diagram, due to the enforced Nishimori symme-

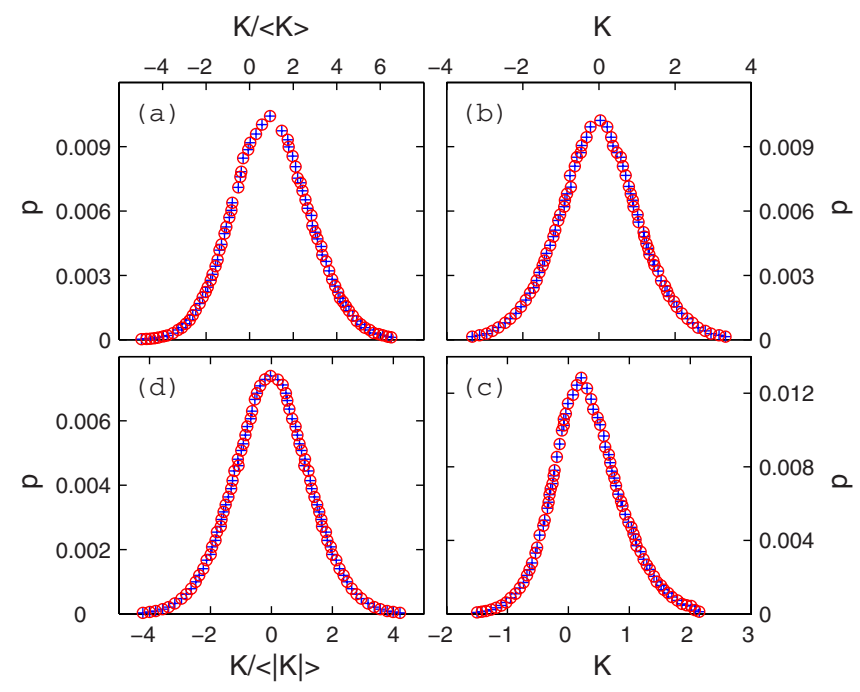

FIG. 7. (Color online) Fixed distributions, with circles and crosses showing one renormalization-group transformation and thereby by their exact superposition attesting to the fixed nature of the distributions. The distributions have been binned for exhibition purposes. (a) For the ferromagnetic-spin-glass phase boundary, a runaway to infinite coupling; (b) for the paramagnetic-spin-glass phase boundary. Both of these fixed distributions are spatially isotropic, attracting isotropic and anisotropic boundaries, and do not obey Nishimori symmetry. (c) For the ferromagnetic-spin-glassparamagnetic multicritical point. This fixed distribution is spatially isotropic and obeys Nishimori symmetry. This fixed distribution attracts the isotropic multicritical point, which obeys Nishimori symmetry, and anisotropic multicritical points, which obey and do not obey Nishimori symmetry. The fixed distributions for the antiferromagnetic-spin-glass, columnar-spin-glass, layered-spinglass phase boundaries and for the antiferromagnetic-spin-glassparamagnetic, columnar-spin-glass-paramagnetic, layered-spinglass-paramagnetic multicritical points are as shown here in (a) and (c), respectively, but with the appropriate $K_{x y} \rightarrow-K_{x y}$ and/or $K_{z} \rightarrow-K_{z}$ reflections. (d) Fixed distribution for the spin-glass phase. This phase sink is an isotropic runaway, attracting both spatially isotropic and anisotropic spin-glass phase points, and does not obey Nishimori symmetry.

try condition $K_{x y}=0$ along the line $p_{x y}=0.5$ and the system reduces to $d=1$. Accordingly, the system is disordered (paramagnetic) along the entire length of this line.

\section{The phase diagram entirely imbedded in Nishimori symmetry}

In the rightmost Fig. 6, both conditions of Eq. (6) are satisfied throughout the figure. With two symmetry constraints, this is a unique surface in the global phase diagram of our model. The system reduces to $d=2$ and $d=1$, as explained above, for $p_{z}=0.5$ and $p_{x y}=0.5$, respectively. The phase boundaries around the paramagnetic phases are actually lines of the multicritical points where the paramagnetic, ordered (ferromagnetic, layered, antiferromagnetic, or columnar), and spin-glass (not seen in this cross section) phases meet.

No spin-glass phase occurs within the Nishimorisymmetric subspace. The phase transitions seen in the right- 
most Fig. 6, namely, ordered-spin-glass-paramagnetic multicritical and ferromagnetic-layered, antiferromagneticcolumnar first-order transitions, are the only phase transitions of the system that occur under Nishimori symmetry.

\section{E. Fixed distributions}

The fixed distributions underpinning the phase diagrams of this system are given in Fig. 7. The fixed distributions for the ferromagnetic-spin-glass boundary, paramagnetic-spinglass boundary, and the ferromagnetic-spin-glassparamagnetic multicritical points are spatially isotropic, but attract both spatially isotropic and anisotropic phase transitions. The fixed distribution for the ferromagnetic-spinglass-paramagnetic multicritical points obeys Nishimori symmetry, but attracts multicritical points that obey and do not obey Nishimori symmetry. In the latter cases, as seen above, both reentrant and forward phase diagrams occur. The fixed distributions for the antiferromagnetic-spin-glass, columnar-spin-glass, layered-spin-glass phase boundaries and for the antiferromagnetic-spin-glass-paramagnetic, columnar-spin-glass-paramagnetic, layered-spin-glassparamagnetic multicritical points are as shown in Figs. 7(a) and $7(\mathrm{c})$, respectively, but with the appropriate $K_{x y} \rightarrow-K_{x y}$ and/or $K_{z} \rightarrow-K_{z}$ reflections.

\section{CONCLUSION}

The exact solution of the spatially uniaxially anisotropic spin glass on a $d=3$ hierarchical lattice yields different phase diagrams. In view of the semiquantitative agreement between spatially isotropic spin-glass results on cubic and hierarchical lattices [19], it would certainly be worthwhile to investigate on cubic lattices the phenomena found in the present study. Furthermore, the exact study of spin glasses on fully anisotropic $d=3$ hierarchical lattices [48] may yield additional phase transition phenomena.

\section{ACKNOWLEDGMENTS}

This research was supported by the Scientific and Technological Research Council (TÜBITTAK) and by the Academy of Sciences of Turkey.
[1] H. Nishimori, Statistical Physics of Spin Glasses and Information Processing (Oxford University Press, Oxford, 2001).

[2] H. Nishimori, J. Phys. C 13, 4071 (1980).

[3] H. Nishimori, Prog. Theor. Phys. 66, 1169 (1981).

[4] H. Nishimori, J. Phys. Soc. Jpn. 55, 3305 (1986).

[5] Y. Ozeki and H. Nishimori, J. Phys. A 26, 3399 (1993).

[6] H. Nishimori, Physica A 205, 1 (1994).

[7] H. Nishimori and K. Nemoto, J. Phys. Soc. Jpn. 71, 1198 (2002).

[8] J.-M. Maillard, K. Nemoto, and H. Nishimori, J. Phys. A 36, 9799 (2003).

[9] K. Takeda and H. Nishimori, Nucl. Phys. B 686, 377 (2004).

[10] K. Takeda, T. Sasamoto, and H. Nishimori, J. Phys. A 38, 3751 (2005)

[11] M. Hinczewski and A. N. Berker, Phys. Rev. B 72, 144402 (2005).

[12] H. Nishimori, J. Stat. Phys. 126, 977 (2007).

[13] K. Binder and K. Schroder, Phys. Rev. B 14, 2142 (1976).

[14] I. Morgenstern and K. Binder, Phys. Rev. Lett. 43, 1615 (1979).

[15] C. Brangian, W. Kob, and K. Binder, J. Phys. A 36, 10847 (2003).

[16] H. G. Katzgraber, M. Körner, and A. P. Young, Phys. Rev. B 73, 224432 (2006).

[17] M. Hasenbusch, A. Pelissetto, and E. Vicari, J. Stat. Mech.: Theory Exp. (2008) L02001.

[18] H. G. Katzgraber, A. K. Hartmann, and A. P. Young, in Computer Simulation Studies in Condensed Matter Physics, Vol. XXI, edited by D. P. Landau, S. P. Lewis, and H. B. Schuttler (Springer Verlag, Heidelberg, 2008), e-print arXiv:0803.3417v1.

[19] G. Migliorini and A. N. Berker, Phys. Rev. B 57, 426 (1998).

[20] F. D. Nobre, Phys. Rev. E 64, 046108 (2001).

[21] M. Ohzeki, J. Phys. Soc. Jpn. 76, 114003 (2007).
[22] M. Hinczewski and A. N. Berker, Eur. Phys. J. B 51, 461 (2006).

[23] M. Hinczewski and A. N. Berker, e-print arXiv:cond-mat/ 0607171v1.

[24] A. N. Berker and S. Ostlund, J. Phys. C 12, 4961 (1979).

[25] R. B. Griffiths and M. Kaufman, Phys. Rev. B 26, R5022 (1982).

[26] M. Kaufman and R. B. Griffiths, Phys. Rev. B 30, 244 (1984).

[27] S. R. McKay, A. N. Berker, and S. Kirkpatrick, Phys. Rev. Lett. 48, 767 (1982).

[28] C. Monthus and T. Garel, Phys. Rev. B 77, 134416 (2008).

[29] A. Falicov, A. N. Berker, and S. R. McKay, Phys. Rev. B 51, 8266 (1995).

[30] E. Domany, S. Alexander, D. Bensimon, and L. P. Kadanoff, Phys. Rev. B 28, 3110 (1983).

[31] J.-M. Langlois, A.-M. S. Tremblay, and B. W. Southern, Phys. Rev. B 28, 218 (1983).

[32] R. B. Stinchcombe and A. C. Maggs, J. Phys. A 19, 1949 (1986).

[33] R. F. Angulo and E. Medina, J. Stat. Phys. 75, 135 (1994).

[34] T. A. S. Haddad, S. T. R. Pinho, and S. R. Salinas, Phys. Rev. E 61, 3330 (2000).

[35] J.-X. Le and Z. R. Yang, Phys. Rev. E 69, 066107 (2004).

[36] B. Derrida and R. B. Griffiths, Europhys. Lett. 8, 111 (1989).

[37] R. A. da Silveira and J.-P. Bouchaud, Phys. Rev. Lett. 93, 015901 (2004)

[38] L.-H. Tang and H. Chaté, e-print arXiv:cond-mat/0007350v1.

[39] C. Monthus and T. Garel, J. Stat. Mech.: Theory Exp. (2008) P01008.

[40] M. Hinczewski and A. N. Berker, Phys. Rev. E 73, 066126 (2006).

[41] M. Hinczewski, Phys. Rev. E 75, 061104 (2007).

[42] Z. Z. Zhang, L. L. Rong, and S. G. Zhou, Physica A 377, 329 (2007). 
[43] Z. Z. Zhang, S. G. Zhou, and T. Zou, Eur. Phys. J. B 56, 259 (2007).

[44] Z. Z. Zhang, Z. G. Zhou, and L. C. Chen, Eur. Phys. J. B 58, 337 (2007).

[45] H.D. Rozenfeld, S. Havlin, and D. ben-Avraham, New J. Phys. 9, 175 (2007)
[46] H.D. Rozenfeld and D. ben-Avraham, Phys. Rev. E 75, 061102 (2007)

[47] E. Khajeh, S. N. Dorogovtsev, and J. F. F. Mendes, Phys. Rev. E 75, 041112 (2007).

[48] A. Erbaş, A. Tuncer, B. Yücesoy, and A. N. Berker, Phys. Rev. E 72, 026129 (2005). 